\title{
La dinámica de una red perinatal del conurbano bonaerense (2008-2017): encuentros entre lo planificado y la práctica
}

\author{
The dynamics of a perinatal network in the greater buenos aires (2008-2017): \\ meetings between planned and practice
}

\author{
Clara Pierini \\ clara.pierini@gmail.com \\ CONICET / Instituto del Conurbano - Universidad \\ Nacional de General Sarmiento, Argentina
}

Recepción: 07 Abril 2019

Aprobación: 22 Junio 2020

Publicación: 02 Agosto 2020

Cita sugerida: Pierini, C. (2020). La dinámica de una red perinatal del conurbano bonaerense (2008-2017): encuentros entre lo planificado y la práctica. Cuestiones de Sociología, 23, e103. https://doi.org/10.24215/23468904e103
Resumen: El artículo analiza la dinámica de una red perinatal regional en el conurbano bonaerense (Provincia de Buenos Aires, Argentina) entre 2008 y 2017.Con este fin, da cuenta de los recorridos de las usuarias por los distintos niveles de la red, e indaga sobre los modos en que se relacionan y articulan los distintos actores involucrados en su atención. Además, analiza los esfuerzos de los niveles locales en la coordinación de las intervenciones orientadas a la atención perinatal, y los mecanismos de referencia y contrarreferencia que se utilizan entre los establecimientos de la red. La estrategia metodológica es cualitativa. Incluyó el relevamiento y sistematización de datos secundarios, y la elaboración y análisis de datos primarios. Finalmente, concluye que los recorridos se ven afectados por la baja articulación entre establecimientos del primer nivel de atención, la convivencia de derivaciones formales e informales, el uso discrecional de los instrumentos de referencia y contrarreferencia, y los obstáculos para derivar pacientes. Estas situaciones dificultan el funcionamiento de la red y se asocian a su informalidad. Sobresale el lugar de los gobiernos municipales y sus profesionales para enfrentar dificultades e intervenir en la organización de la red y su dinámica.

Palabras clave: Redes de servicios de salud, Atención perinatal, Conurbano bonaerense, Recorridos de las usuarias, Coordinación de la atención sanitaria.

Abstract: The article analyzes the dynamics of a regional perinatal network in the Greater Buenos Aires (Buenos Aires Province, Argentina) between 2008 and 2017. For this purpose, it presents the users' journeys through the network's different levels, and digs into the relationship and articulation between the diverse actors involved in their attention. In addition, it analyzes the efforts of the local levels in the coordination of the interventions oriented to perinatal care, and the referral and counter-referral mechanisms that are used among the network's institutions. The methodological strategy is qualitative. It included the survey and systematization of secondary data, and the elaboration and analysis of primary data. Finally, it concludes that user experiences are affected by the low articulation between institutions at the first level of care, the coexistence of formal and informal referrals, the discretionary use of referral and counter-referral instruments, and obstacles to refer patients. These situations hinder the operation of the network and are 
associated to its informality. The role of municipal governments and their professionals to face difficulties and intervene in the network's organization and dynamics is very prominent.

Keywords: Health service networks, Perinatal care, Greater Buenos Aires, Users' journeys, Coordination of health care.

\section{INTRODUCCIÓN}

El artículo atiende a las dinámicas de una red perinatal regional del conurbano bonaerense ${ }^{1}$ (Región Sanitaria VII). Con este propósito, da cuenta de los recorridos y el tránsito de las usuarias ${ }^{2}$ por los distintos niveles de la red, e indaga sobre los modos en que se relacionan, articulan e intercambian los actores involucrados en su atención. Además, analiza los esfuerzos de los niveles locales de gobierno en la coordinación de las intervenciones orientadas a la atención perinatal. Por último, devela los particulares mecanismos de referencia y contrarreferencia que se utilizan entre los establecimientos al interior de la red, y muestra sus alcances en el territorio.

Esta propuesta pone énfasis en las tensiones y negociaciones inevitables que aparecen en el devenir de un trabajo articulado, como en las salidas negociadas para su resolución. En el caso elegido (como consecuencia del contexto, los atributos del subsector sanitario estatal en el conurbano y las características de las trayectorias en busca de atención perinatal), las "soluciones" a veces se alcanzan de manera formal, mediante contratos o normativas, y otras veces a través de vías informales.

\section{LA "CAJA DE HERRAMIENTAS": LINEAMIENTOS TEÓRICO-CONCEPTUALES PARA PENSAR LAS REDES PERINATALES Y LOS RECORRIDOS DE LAS USUARIAS Y SU SEGUIMIENTO}

\section{Los conceptos de "redes de servicios de salud" y "redes perinatales"}

Las propuestas interesadas por la aplicación del "modelo de redes" (redes de políticas) al campo de la planificación y gestión en salud, manifiestan que las redes involucran una articulación de organizaciones (estatales, privadas, sociedad civil) que ofrecen un continuo coordinado e integral de servicios sanitarios para una población y territorio específicos. Además, deben hacerse cargo de los costos y resultados (clínicos, económicos y del estado de salud de la población), y rendir cuentas por ellos. En América Latina, las redes de servicios de salud desarrolladas en el subsector público se implementaron como una estrategia para garantizar el acceso de la población a la atención integrando los distintos niveles de complejidad. Se organizan a partir de la Atención Primaria de la Salud, y sostienen modelos que concentran ciertos servicios (hospitales, centros especializados) y favorecen la dispersión de otros (centros de salud) (Vilaça Mendes, 2013; Ariovich y Crojethovic, 2015).

Las redes de servicios de salud orientadas a la atención perinatal se caracterizan por la organización de sus servicios de manera jerarquizada y según niveles de complejidad creciente. Cada una de sus instituciones tiene un nivel de complejidad particular, alcanzado mediante acuerdos entre los establecimientos y equipos de salud, y en relación a las necesidades de la población. Estas redes se organizan en tres niveles de atención, que presentan algunas especificidades de acuerdo a su población objetivo (las mujeres gestantes y puérperas, y los recién nacidos), y a los requerimientos que supone la atención materna, fetal y neonatal de calidad (un primer nivel fuerte y capacitado para asignar a las usuarias el nivel de atención que les corresponda en relación al riesgo; buenos medios de comunicación; y un sistema de traslados organizado y centralizado). Su organización en tres niveles que trabajan de modo articulado y en red propicia la conformación de "corredores 
sanitarios" (Ministerio de Salud de la Nación, 2010; Schwarcz, 2011; Speranza y Kurlat, 2011; Simioni et al., 2017).

El primer nivel de atención se encarga, a través de los servicios de obstetricia y neonatología y de modo ambulatorio, del control de embarazadas, de la detección del riesgo obstétrico y del control del recién nacido sano. El segundo y el tercer nivel refieren a establecimientos hospitalarios con capacidad para realizar partos. En la categorización de los servicios de maternidad, la diferencia central es la presencia o no de terapia intensiva de adultos u obstétrica; los servicios de neonatología se categorizan según la edad gestacional (OPSMSAL, 2011; Fariña, 2018). Es decir, los establecimientos del segundo nivel sólo se ocupan delos embarazos "bajo riesgo" y los hospitales del tercer nivel poseen mayor complejidad.

\section{Algunos conceptos relativos al tránsito, seguimiento y coordinación en la atención}

Para describir y analizar las dinámicas de la red, es preciso presentar algunas nociones. En primer lugar, el concepto de acceso, que permite reflexionar acerca de las debilidades y fortalezas de la red estudiada. Esta noción (centrada en las personas) entiende que los individuos, incluso cuando viven en un mismo lugar y comparten características socioeconómicas, pueden tener acceso diferencial a lugares, actividades, bienes y servicios. El acceso incluye las distancias materiales y el transporte, pero también depende de aspectos personales y familiares, de la actividad o servicio que se busca satisfacer (adquiere particularidades en la búsqueda de atención sanitaria) y del contexto sociohistórico (Gutiérrez, 2011).

Las nociones de continuidad y coordinación, en su aplicación al campo de la salud, se encuentran estrechamente vinculadas al acceso, ya que inciden de manera positiva o negativa sobre el mismo. La continuidad de la atención remite a la existencia de una relación perpetua y duradera entre las personas usuarias y el equipo de salud o establecimiento, que promueve relaciones basadas en la confianza y el conocimiento cercano. Garantiza una atención longitudinal y basada en la persona (no en la enfermedad) (Ariovich y Jiménez, 2009; Berra et al., 2011; Ariovich, 2015).

La coordinación refiere al enlace entre los servicios o establecimientos en vistas de que los usuarios reciban la atención y seguimiento adecuados en relación al evento o problema de salud que enfrenten. Esta característica es imprescindible para garantizar la integración y continuidad en la atención entre los distintos niveles de atención, e incluye la referencia, el acompañamiento y la contrarreferencia. Además, supone que el profesional conoce los problemas o eventos que con anterioridad atravesó la paciente, como los servicios por él utilizados (Ariovich y Jiménez, 2009; Berra et al., 2011; Ariovich, 2015).

El concepto de referencia remite específicamente al envío de usuarias o elementos de ayuda diagnóstica por parte de un establecimiento sanitario a otra institución (del sector salud) de mayor complejidad para la atención o complementación diagnóstica. La contrarreferencia, por su parte, es la respuesta de la institución que recibe la referencia a la unidad que la demanda o al usuario. Así, supone la contrarremisión del paciente una vez definido el diagnóstico o tratamiento (con indicaciones a seguir, para su control y seguimiento) o el envío de los resultados solicitados. En ambos casos, se trata de procedimientos administrativos formales (Ariovich y Jiménez, 2014, p. 52).

Con la intensión de ahondar sobre el lugar de los mecanismos de referencia y contrarreferencia y los protocolos en el trabajo de la red y, particularmente, respecto de en qué medida lograron a través de la regulación de relaciones alcanzar transformaciones en el modo de organizar la atención (Chiara, 2018), se recuperan los aportes de Lascoumes y Le Galés (2007) sobre los instrumentos de políticas. Estos autores destacan que los instrumentos permiten acercarse a las técnicas y usos que estructuran las políticas y, en consecuencia, los definen como dispositivos técnico-sociales que organizan las relaciones entre el poder público y los beneficiarios. 


\section{MODO DE ABORDAJE}

\section{Recorte espacio-temporal}

Se seleccionó una región sanitaria como unidad de estudio ya que, en la Provincia de Buenos Aires, las regiones sanitarias constituyen espacios descentralizados del Estado provincial en materia de salud, encargados de la planificación, gestión y/o articulación de políticas sanitarias desde una mirada regional. Como explican Alonso, Gutiérrez y Merlinsky (2016, p. 490), una región sanitaria está compuesta por varios municipios y define la escala territorial dentro de la cual se brindan recursos y se organizan la infraestructura y los programas relativos a las prestaciones de salud. Cada región tiene un régimen de referencia y contrerreferencia entre establecimientos (ya sean nacionales, provinciales y municipales) y opera como espacio de articulación interjurisdiccional.

La elección de la Región Sanitaria VII responde a intereses que exceden los fines del artículo (se inserta en la investigación de maestría de la autora). Sucintamente, resulta de interés como caso porque posee los siguientes atributos: buena presencia de establecimientos de los tres niveles de atención, la existencia de efectores de las tres jurisdicciones (Nación-Provincia-municipios), el funcionamiento de un hospital de la más alta complejidad (Hospital Posadas) y la implementación del proceso de "Regionalización de la Atención Perinatal” (RAP). Además, presenta alta densidad poblacional y un elevado porcentaje de nacimientos sobre el total del conurbano. 5

La RAP fue una estrategia promovida a partir del 2010 por un plan nacional ("Plan Operativo para la Reducción de la Mortalidad Materno Infantil, de las Mujeres y de las Adolescentes”) que constituyó un elemento clave para el desarrollo de redes perinatales (con una estructura, organización y dinámica singulares). En este marco, en el conurbano, la Nación promovió una restructuración de la atención perinatal según niveles de complejidad, que fue llevada adelante por el gobierno provincial y asignó un lugar central a las regiones sanitarias.

La Región Sanitaria VII está compuesta por los municipios de General Las Heras, General Rodríguez, Luján, Marcos Paz, Merlo, Hurlingham, Ituzaingó, Morón, Tres de Febrero y Moreno. En el presente trabajose consideranlos municipios que pertenecen al conurbano, dado las particularidades de su territorio (particularmente, la continuidad de la trama urbana y la cantidad de población) ylos municipios de General Las Heras, General Rodríguez, Luján y Marcos Paz fueron excluidos. La Región VII tiene una población de 2.253.772 habitantes y los seis partidos bajo estudio alcanzan los 1.991 .244 habitantes, el 19,41\% de la población del conurbano (Censo 2010).

Se considera el periodo comprendido entre 2008 y 2017. Inicia con la incorporación de la Provincia de Buenos Aires al "Plan Nacer" (programa implementado por la cartera sanitaria nacional, que refiere a seguros públicos de salud provinciales para la población materno-infantil sin obra social) e incluye el inicio del proceso de RAP.

\section{Estrategia metodológica}

La estrategia metodológica es cualitativa e incluyó el relevamiento y sistematización de datos secundarios y la elaboración y análisis de datos primarios. La búsqueda de información secundaria consideró el relevamiento, por un lado, de normativa vinculada a la gestión de la atención perinatal en sus distintos niveles (nacional, provincial y municipal) y a la organización de los servicios de salud perinatal y, por el otro, de documentos de programas nacionales y provinciales referidos a la atención materno-infantil (por ejemplo: "Plan Operativo para la Reducción de la Mortalidad Materno Infantil, de las Mujeres y de las adolescentes"; Plan Nacer; Programa "Al fin en casa"6). Además, involucró la sistematización de informes 
de gestión, convenios, protocolos y guías de procedimientos específicos a la red perinatal de la Región Sanitaria VII.

La realización de entrevistas semi-estructuradas a informantesvinculados a la gestión de la atención perinatal en las distintas jurisdicciones (Nación, Provincia y municipios) y a profesionales de los establecimientos, buscó reconstruir y analizar la experiencia de la red. Las entrevistas permitieron caracterizar la estructura, dinámica y alcance de la red, atendiendo a sus actores y a las relaciones interinstitucionales.

Se realizaron veintinueve entrevistas entre junio de 2017 y octubre de 2018 (Tabla 1). La muestra comprendió representantes de los distintos niveles de gestión que participan en la red; además, en el nivel local, incluyó a profesionales de todos los municipios considerados. Se entrevistó a personas con diversos cargos, actividades y profesiones, y se consideró que el entrevistado o su lugar de trabajo tuvieran un rol clave en la red. Las interlocutoras fueron puestas al corriente de la investigación; también, se les solicitó su consentimiento y se les garantizó confidencialidad. El análisis de las entrevistas fue realizado con la ayuda del software Atlas Ti.

Tabla 1. Entrevistas realizadas, junio 2017- octubre 2018.

\begin{tabular}{|c|c|c|c|}
\hline \multicolumn{4}{|c|}{ RegiónSanitariaVII } \\
\hline & Macro-gestión & Meso-gestión & Micro-gestión \\
\hline & $\begin{array}{l}\text { 10 } \\
\text { 1. FuncionariadelMinisteriodeSal } \\
\text { uddelaProvinciadeBuenosAires, } \\
\text { referenteeneläreamaterno- } \\
\text { infantil [2007-2015] }\end{array}$ & $\begin{array}{l}\text { TD } \\
\text { 12. DirectoradeMaternidadprovinc } \\
\text { ial [2015-2017] }\end{array}$ & $\begin{array}{l}\text { 19. ProfesionaldelAreadeOb } \\
\text { stetriciadehospitalnacional[ } \\
\text { Todoelperiodoestudiado] }\end{array}$ \\
\hline & $\begin{array}{l}\text { TD } 2 . \\
\text { Referenteprogramaprovincialori } \\
\text { entadoaladisminucióndelamorta } \\
\text { lidadmaterna [2016-actualidad] }\end{array}$ & $\begin{array}{l}\text { TD } \\
\text { 13.Profesionales deláreadegestió } \\
\text { ndeMaternidadprovincial [2013- } \\
\text { actualidad] }\end{array}$ & $\begin{array}{l}\text { TD } \\
\text { 20. Médicadecentrodesalud } \\
\text { municipal [2012-actualidad] }\end{array}$ \\
\hline & $\begin{array}{l}\text { 7D } \\
\text { 3.Referentedeprogramaprovinci } \\
\text { alorientadoalaasistenciadelosre } \\
\text { ciênnacidosprematurosysusfami } \\
\text { lias [2016-actualidad] }\end{array}$ & $\begin{array}{l}\text { 10 } \\
\text { 14. DirectoradeCentrodeSaludde } \\
\text { municipal[Todoelperiodobajoestu } \\
\text { dio] }\end{array}$ & $\begin{array}{l}\text { 21. JefadeServiciodeObstetr } \\
\text { iciadeMaternidadprovincial } \\
\text { [2017-actualidad]. }\end{array}$ \\
\hline & $\begin{array}{l}\text { TD } \\
\text { 4. FuncionariadelMinisteriodeSal } \\
\text { uddelaNación (MSAL), } \\
\text { referenteeneläreamaterno- } \\
\text { infantil [2009-2015] }\end{array}$ & $\begin{array}{l}\text { 7D } \\
\text { 15. ReferentedeláreadeMaternida } \\
\text { delnfanciadelaSecretariadeSalud } \\
\text { municipal[Todoelperiodobajoestu } \\
\text { dio] }\end{array}$ & $\begin{array}{l}\text { 22. ProfesionaldelAreadeOb } \\
\text { stetriciadehospitalprovincial } \\
\text { [Todoelperiodobajoestudio] }\end{array}$ \\
\hline & $\begin{array}{l}\text { TD } \\
\text { 5.ProfesionaldelaDirecciónMate } \\
\text { rnidadel InfanciadelMSAL. } \\
\text { [Todoelperiodobajoestudio] }\end{array}$ & $\begin{array}{l}\text { TD } \\
\text { 16. DirectoradeCentrodeSaludmu } \\
\text { nicipal[Todoelperiodobajoestudio } \\
\text { ] }\end{array}$ & $\begin{array}{l}\text { 1D } \\
\text { 23. ProfesionaldelAreadeNe } \\
\text { onatologiadehos pitalnacion } \\
\text { al.[Todoelperiodobajoestudi } \\
\text { o] }\end{array}$ \\
\hline & $\begin{array}{l}\text { TD } \\
\text { 6.Referentedelal niciativaMatern } \\
\text { idadSegurayCentradaenlaFamil } \\
\text { iaUNICEF } \\
\text { [Todoelperiodobajoestudio] }\end{array}$ & $\begin{array}{l}\text { 7D } \\
\text { 17.ReferentemunicipaldeObstetri } \\
\text { cia[Todoelperiodobajoestudio] }\end{array}$ & $\begin{array}{l}\text { 24. JefedeServiciodeNeonat } \\
\text { ologiadehos pitalmunicipal } \\
\text { [2010-2017]. }\end{array}$ \\
\hline & $\begin{array}{l}\text { 7. FuncionariadeSecretariadeSa } \\
\text { ludmunicipal, } \\
\text { referenteeneläreamaterno- } \\
\text { infantil[2016-actualidad] }\end{array}$ & $\begin{array}{l}\text { T1D } \\
\text { 18. DirectorCentrodeSaludmunici } \\
\text { pal[2012]. }\end{array}$ & $\begin{array}{l}\text { TD } \\
\text { 25. ProfesionaldelAreadeOb } \\
\text { stetriciadehospitalmunicipal } \\
\text { y centro de salud } \\
\text { municipal[Todoelperiodobaj } \\
\text { oestudio] }\end{array}$ \\
\hline & $\begin{array}{l}\text { 1D } \\
\text { 8.FuncionariodeSecretariadeSa } \\
\text { ludmunicipal [Hasta 2015] }\end{array}$ & & $\begin{array}{l}\text { ID } 26 . \text { Lic. } \\
\text { enObstetriciadeCentrodeSa } \\
\text { ludmunicipal[Todoelperiodo } \\
\text { bajoestudio] }\end{array}$ \\
\hline & $\begin{array}{l}\text { ID } \\
\text { 9.ReferentedelaestrategiadeRe } \\
\text { gionalizacióndelaAtenciónPerin } \\
\text { ataldelMSAL } \\
\text { [Todoelperiodobajoestudio]. }\end{array}$ & & $\begin{array}{l}\text { 1D } \\
\text { 27. ProfesionaldelServiciode } \\
\text { Obstetriciadehos pitalprovin } \\
\text { cial[Todoelperiodobajoestu } \\
\text { dio] }\end{array}$ \\
\hline & $\begin{array}{l}\text { 10. FuncionariodeSecretariadeS } \\
\text { aludmunicipal, } \\
\text { referenteenAtenciónPrimariadel } \\
\text { aSalud [2008 - 2014] }\end{array}$ & & $\begin{array}{l}\text { ID 28. Lic. } \\
\text { enObstetriciaencentrodesal } \\
\text { udmunicipal[Todoelperiodo } \\
\text { bajoestudio] }\end{array}$ \\
\hline & $\begin{array}{l}\text { 1D } \\
\text { 11. FuncionariadelaRegiónSanit } \\
\text { ariaVII, referenteeneláreaperinat } \\
\text { al [Todoelperiodobajoestudio] }\end{array}$ & & $\begin{array}{l}\text { 10 29.Lic. } \\
\text { enTrabajoSocialencentrode } \\
\text { saludmunicipal [2014-2017] }\end{array}$ \\
\hline
\end{tabular}

Fuente: Elaboración propia 


\section{La estructura y organización dela Red perinatal de la Región Sanitaria VII}

La red perinatal de la Región Sanitaria VII posee tres niveles de atención con características particulares dado su inserción en territorios loco-regionales específicos, como por la participación de establecimientos de diversas dependencias con lógicas de trabajo singulares (Pierini, 2019).

$\mathrm{Al}$ primer nivel de atención lo conforman todas postas sanitarias, centros de atención primaria de la salud (CAPS) y centros de integración comunitaria (CIC $)^{7}$ de dependencia municipal, que suman alrededor de 113 en la Región analizada (Mapa 1).En este nivel se realizan los controles prenatales, puerperales y del recién nacido; además, se detecta el riesgo obstétrico y los embarazos de alto riesgo son derivados al hospital de referencia del partido. Su adaptación a las necesidades de la práctica depende notablemente de los esfuerzos y la creatividad de las/os profesionales (por ejemplo: se observa que en los CAPS varían las especialidades a cargo de los controles prenatales, y la oferta y acceso a controles puerperales) (Pierini, 2019).

Respecto al segundo y tercer nivel, la red está compuesta por un hospital nacional, cinco hospitales provinciales y cuatro hospitales municipales. El Mapa 1 muestra que la presencia de maternidades varía según el municipio, como así también la complejidad disponible. ${ }^{8}$ En Moreno hay dos establecimientos hospitalarios de dependencia provincial: la Maternidad Estela de Carlotto, de nivel II, y el Hospital Descentralizado Zonal General Mariano y Luciano de la Vega, de nivel IIIB. En Merlo se radican tres hospitales con servicios de maternidad, dos de ellos de dependencia municipal y de nivel II (Hospital Materno Infantil Pontevedra y Hospital Materno Infantil Dr. Chutro), y un hospital provincial de nivel IIIB (Hospital Zonal General de Agudos Héroes de Malvinas). Ituzaingó carece de hospitales en su jurisdicción. Morón posee dos maternidades en su territorio, ambas de nivel IIIB, una en el Hospital Nacional Dr. A. Posadas y otra en el Hospital Municipal Ostaciana B. de Lavignolle. En Hurlingham, el Hospital Municipal San Bernardino de Siena posee una una maternidad nivel II. Por último, en Tres de Febrero se ubican dos maternidades provinciales: una de nivel IIIA (Hospital Zonal General de Agudos Dr. Carrillo) y otra de nivel IIIB (Hospital Zonal General de Agudos Dr. Bocalandro) (MS-PBA, Programa de apoyo a la inversión en los sectores sociales de la Provincia de Buenos Aires, 2016; MEC, 2017). 
Mapa 1. Establecimientos que conforman la red perinatal de la

Región Sanitaria VII según ubicación y nivel de complejidad.

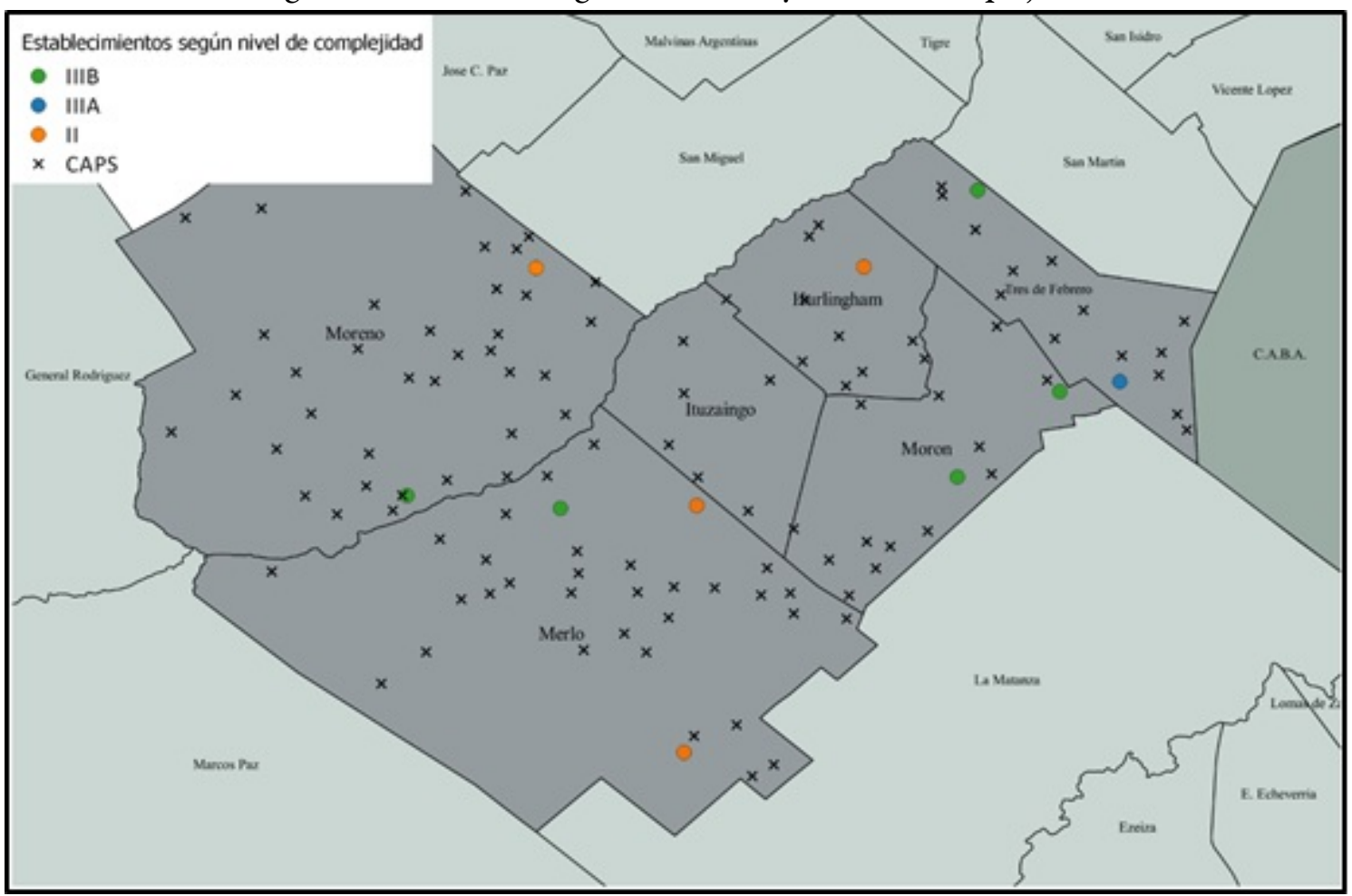

Fuente: Elaboración propia sobre la base de datos del Ministerio de Salud de la provincia de Buenos Aires (MSP, 2012; DIS-MSP, 2015) y de las Secretarias de Salud municipales (Municipalidad de Ituzaingó, 2014; Gobierno de Morón, s.f.; Municipio de Tres de Febrero, 2018; Municipio de Merlo, s.f.; Municipio de Moreno, 2018).

Respecto al funcionamiento del segundo y tercer nivel, vale realizar algunas aclaraciones. Si bien el proceso de categorización y acreditación de establecimientos hospitalarios con servicios de maternidad, en el marco de la RAP, asignó un nivel y tareas a cada uno de ellos y tuvo un rol saliente en la organización de la red, no saldó los conflictos que se dan en la labor cotidiana. El trabajo de campo evidenció que los actores intervinientes, muchas veces, desconocen el nivel de otros establecimientos involucrados e, incluso, del servicio en donde trabajan; además, mostró dificultades en la referencia y contrarreferencia. La iniciativa de categorización y acreditación da cuenta de la participación y acatamiento de los niveles provincial y municipal (y de sus hospitales) a los lineamientos nacionales, aunque las entrevistas indican que en la práctica se trabaja según lógicas y ordenamientos que no responden necesariamente a estos (Pierini, 2019).

Por otra parte, además de ocuparse de las tareas que corresponden a su nivel, todos los hospitales de la red poseen consultorios para el control de embarazos de bajo riesgo (con excepción de la Maternidad Carlotto), aunque de acuerdo a las recomendaciones nacionales esta es una competencia del primer nivel (Pierini, 2019).

\section{LA DiNÁMICA DE LA RED: ENCUENTROS ENTRE LO PLANIFICADO Y LA PRÁCTICA}

\section{Los caminos de las personas gestantes a través de los servicios9}

Según el relato de trabajadores de distintos niveles de gestión, la red perinatal comienza en el barrio. La puerta de entrada son los centros de atención primaria de la salud (CAPS), a donde las mujeres llegan para iniciar los controles prenatales. 
Generalmente las embarazadas o se acercan al centro de atención primaria, piden turno porque ya saben, lo conocen o se referencian, o vienen al Hospital y piden turno en los consultorios. Lo que están haciendo muchas obstétricas es tratar de referenciarlas (...) con algún centro de salud que les quede más cerca, porque todavía por ahí circula esto entre las mujeres que si no se controlan el embarazo en el hospital, cuando vengan a parir no van a tener su cama. [ID 25]

Si bien la búsqueda activa y el fortalecimiento del trabajo de los promotores de salud aparece como un ideal o deber, el trabajo de campo no indica que sea una actividad fortalecida en la labor del primer nivel, lo que incide en el acceso a sus servicios. Algunos entrevistados explicaron que la falta de profesionales y promotores atenta contra el cumplimiento de estas tareas. En general, las mujeres que se acercan para controles prenatales ya conocen el centro de salud y acuden por iniciativa propia.

Por otra parte, hay usuarias que inician sus recorridos en el segundo y tercer nivel, como es el caso de quienes deciden controlar allí sus embarazos de bajo riesgo. La modalidad de ingreso a la red le imprime al recorrido de la persona gestante particularidades, especialmente en términos de acceso (los CAPS se caracterizan por su proximidad y un abordaje de la salud amplio y centrado en el grupo familiar). También, da cuenta de variaciones respecto de los caminos formalmente delineados para la atención perinatal (a nivel nacional, provincial y municipal); por ejemplo: los materiales y guías del municipio de Moreno indican que el camino de la embarazada comienza en el CAPS, donde se controlan los embarazos hasta la semana 36, pero los consultorios de bajo riesgo siguen funcionando en los hospitales que forman parte de su corredor perinatal (Hospital M. y L. de la Vega y Hospital Posadas) (MEC, 2017).

La modalidad de asignación de turnos para controles prenatales carece de criterios regionales o locales (como los tiempos de espera, los requisitos y la facilidad/dificultad para obtenerlos), varía incluso entre CAPS de un mismo municipio y hospitales con la misma dependencia. Así, se presentan algunas citas ilustrativas de situaciones relevadas en el primer nivel, que resultan relevantes por ser el nivel institucionalmente definido para el control de embarazos de bajo riesgo. Los ejemplos muestran que los profesionales tienen influencia en el modo en que se organiza el servicio y dan cuenta de la crítica situación en materia de recursos humanos de algunos municipios.

Acá yo, particularmente, llegan y las atiendo por orden de llegada, no tienen que sacar turno ni venir antes, nada. (...) porque no tengo tantas pacientes. Allá [en otro CAPS del municipio] sí daba turnos, igual atendía por turnos y después atendía a todas las que venían; pero acá es demanda espontánea. (...)igual tengo una libretita ahí donde me las cito, viste, porque el control está como protocolizado. [ID 26]

El único sistema actualmente es a demanda: las mujeres vienen y demandan el turno, siempre se trata de priorizar, pero la realidad es que muchas mujeres [embarazadas] quedan afuera. No es que quedan afuera, sino que tienen turnos de acá a dos o tres meses. (...) En realidad el sistema se trata de pensar siempre en ubicarlas. Pero están las obstétricas desbordadas, los médicos también. [ID 20]

Por otro lado, en relación a los estudios complementarios solicitados durante los controles prenatales, tampoco existe un paquete definido que brinden los CAPS, ni una sola modalidad de acceder a los turnos (ya sea que se realicen en el primer nivel o en hospitales). En los CAPS de Moreno se asignan turnos para hacer los laboratorios y ecografías allí mismo (ID 16); en Tres de Febrero las embarazadas tienen que acerca otro día para realizarlos (ID 26); y en Ituzaingó se realizan sólo en el CAPS más grande y con mayor número prestaciones con un turno programado que brinda la obstétrica en el control (ID 17). Además, estos turnos pueden agilizarse según el caso y el profesional que intervenga, por la vía institucional (en general mediante alguna dependencia de la secretaría de salud municipal) o por la informal. Una profesional del primer nivel, que además se desempeña en un hospital municipal, indicó que a veces les decía a las usuarias: "Vení a verme tal día al Hospital y repetimos un laboratorio, hacemos esto, te contacto" (ID 25).

Respecto a las articulaciones entre establecimientos del primer nivel aparecen algunas menciones sobre reuniones de directores de CAPS y de las redes de obstétricas municipales, como algunos intercambios entre establecimientos asociados a la proximidad, la necesidad de seguimiento de usuarios o la redistribución de medicamentos; pero las referencias no abundan. Algunos profesionales de este nivel explican la dificultad 
de solicitar derivaciones para controles y estudios a otros CAPS, ya que todos se encuentran en las mismas condiciones (faltan profesionales y hay más trabajo del que pueden abordar). Así, es posible sostener la escasa articulación entre establecimientos del primer nivel al interior de la red; las articulaciones son mayormente entre CAPS y establecimientos del segundo y tercer nivel con la intención de referenciar usuarias para su atención con una mayor complejidad.

En todos los municipios, el camino que las embarazadas iniciaron en el primer nivel continúa en un hospital o maternidad. Allí la usuaria se dirige, con indicación de un profesional, porque su embarazo reviste cierta complejidad y precisa ser controlado en un consultorio de alto riesgo, o porque se encuentra cerca del momento del parto.

El acceso a los consultorios de alto riesgo o a especialidades (cardiología, diabetología, entre otras) dentro de los establecimientos de mayor complejidad presenta variaciones. De acuerdo al caso, después de la consulta en una especialidad, las mujeres pueden continuar controlándose en el primer nivel o en el consultorio de bajo riesgo del hospital. Sobresale que algunas entrevistadas del primer nivel comentan que, aunque deriven los casos de alto riesgo al hospital, las mujeres son re-citadas; ya que el vínculo que aquí se establece con los usuarios es de mayor proximidad y no sólo atiende a cuestiones biomédicas (sino también sociales, de género, etc.), lo que garantiza mayor adherencia. Incluso una profesional mencionó que: "Muchas veces son derivadas a alto riesgo por una cuestión legal"(ID 16).

Sobresale la variedad de situaciones posibles y elecciones a la hora de referenciar a una usuaria a un hospital (en busca de mayor complejidad o por la proximidad del parto). Algunas de ellas revisten notable formalidad, por ejemplo entre CAPS y la Maternidad Carlotto en Moreno o entre CAPS y Hospital Lavignolle en Morón; otras se sostienen en la informalidad. Los CAPS no necesariamente referencian a las usuarias a un establecimiento de nivel II, ya que no existen establecimientos de este nivel en todas las jurisdicciones y los caminos institucionales suponen la derivación de la mujer al hospital localizado en el municipio donde se atiende, aunque también pesan otras cuestiones. Muchas veces (incluso cuando existen caminos delineados formalmente) la elección depende del criterio del profesional interviniente y sus vínculos personales, como de la mujer y sus posibilidades en términos de acceso.

En los casos donde aparecen complicaciones, algunos entrevistados han mencionado derivar a la usuaria a un establecimiento donde se trata su patología, ya que consideran que allí se trabaja bien o poseen algún contacto informal. Una obstétrica del primer nivel de Tres de Febrero mencionó que, aunque los Hospitales Bocalandro y Carrillo sean los de referencia municipal, ella deriva a las mujeres para el momento del parto donde deseen y les quedé más cómodo (con una orden de derivación con sus datos); también indicó que, en caso de solicitarles estudios, a veces contacta a alguien que conozca en los hospitales del distrito para evitar que se pierdan o las deriva a un establecimiento de la Ciudad de Buenos Aires, donde se desempeña y accede a turnos con facilidad (ID 26). También, en Moreno, una profesional indicó que cuando se presentan situaciones de alto riesgo, si lo considera mejor, referencia a las pacientes directo al Hospital Posadas o a establecimientos por fuera del corredor a través de vínculos personales(ID 16). Respecto del caso del corredor de Moreno, algunos profesionales prefieren al Hospital L. y M. de la Vega (de mayor complejidad) por sobre la Maternidad Carlotto, e indican que esta Maternidad resulta inaccesible para muchas morenenses (que tienen que tomarse dos colectivos, lo que supone una inversión de tiempo y dinero). En Morón, también se han mencionado situaciones en las que las obstétricas derivan a las mujeres, si estas pueden acudir, directo al Hospital Posadas.

Se destaca el caso de Ituzaingó, municipio que carece de maternidad y donde (con intervención de la Región Sanitaria VII) se han armado recorridos "artesanales" para asegurar a sus usuarias la continuidad de la atención. Si bien estas estrategias no son formales, se encuentran institucionalizadas.

Con el trabajo de la Región Sanitaria, empezamos a tener vínculos con los distintos efectores de los municipios vecinos (...) No hay ningún convenio oficial. No es que: «Me la va a atender fulanito de tal Hospital». Pero yo ya sé que al[Hospital] Chutro, si la mando a las 35 semanas con la última fotocopia, con el papelito firmado, con el plancito Nacer y no sé qué, la 
atienden. En la[Maternidad] Carlotto no, tiene que ir con tal y tal condición a tal semana. En el[Hospital] Posadas tampoco, tiene que ir. Entonces, depende la sala a donde estés, el hospital que te queda más cerca conocemos cuáles son los requisitos para que la paciente pueda ir a atenderse sin problema. Y hacemos medio como algo artesanal, cuando llegan los 7 meses le preguntamos: «¿cuál es tu idea?» (...) también depende de los profesionales. Por ejemplo, el doctor que hace alto riesgo, es el jefe de alto riesgo de Morón. Se las autoderiva. Él cuando ve que acá ya no da para más o que necesita internación o lo que fuere, se la lleva a Morón, no la manda al Posadas. [ID 17].

Todos los establecimientos de segundo y tercer nivel de la red perinatal de la Región Sanitaria VII reciben personas en trabajo de parto que se acercan por elección propia; luego de haber "rebotado" en otro hospital (por falta de camas, deficiencias edilicias o falta de profesionales) "y no les queda otra"; derivadas formalmente desde el primer nivel u otros hospitales (por las mismas causas que "rebotan" o por alguna situación vinculada al riesgo obstétrico).

Derrotero del Oeste significa la paciente que llega a las 4 de la mañana y salió de su casa a las 8 de la noche; que fue al [Hospital] Chutro, que no tenía Neo; que fue al [Hospital] Carrillo, que no tenía Obstetricia; que fue al [Hospital Héroes de] Malvinas y no tenía esto, y cayó al [Hospital] Posadas; caminando, en colectivo, en auto [ID 19].

También están quienes ingresan a través de la Guardia, las Unidades de Pronta Atención (UPA) o el Servicio de Emergencias.

Luego del parto, el camino de las embarazadas sigue en el primer nivel (con excepción de las mujeres con partos por cesárea o recién nacidos prematuros, que continúan los controles en el hospital). No todas las usuarias regresan para el control puerperal a los CAPS. Las obstétricas muestran preocupación por las cuestiones que se abordan en esta consulta ${ }^{10}$ y porque no pueden cerrar la historia perinatal (necesaria para la carga del Sistema Informático Perinatal ${ }^{11}$ ). En el próximo apartado se destacan algunas experiencias que denotan la iniciativa y creatividad del nivel municipal, como la dificultosa coordinación de acciones entre establecimientos.

El acceso para controles prenatales, estudios, especialidades y consultorios de alto riesgo (tanto en CAPS como en establecimientos de segundo y tercer nivel) presenta dificultades, particularmente si se consideran las características sociosanitarias de la población que utiliza el subsector público en la Región VII. ${ }^{12}$ Estas barreras, además, persisten al momento del parto. La realización de entrevistas a actores de la meso y micro-gestión y, en particular, la indagación sobre prácticas e intervenciones concretas, permitió reconocer las negociaciones y arreglos para coordinar acciones entre niveles de atención y de gobierno y garantizar trayectorias de continuidad (no siempre con éxito). Estas negociaciones y arreglos refieren, especialmente donde no están delineados corredores formales (pero allí también), a soluciones alcanzadas de modo informal a través de vínculos personales, pedidos de favores y buena voluntad. Las entrevistas dan cuenta de que cuando no interviene ningún establecimiento/profesional, o los arreglos informales no resuelven el paso al siguiente nivel de la red, los usuarios (muchas veces) atraviesan el "derrotero de la VII", experiencia que pone de manifiesto las grandes limitaciones para garantizar trayectorias de continuidad como también la falta de coordinación formal entre niveles. Más adelante, se indican algunos esfuerzos y dispositivos que buscan dinamizar y facilitar las trayectorias de las mujeres, y se mencionan ciertos instrumentos de los que se valen las redes para fortalecer/transformar el modelo de atención.

Los recorridos presentados (que muestran preferencias, idas y vueltas, intervenciones de diversos actores, establecimientos valorizados y no valorizados) asignan al territorio atributos específicos y confirman la necesidad de considerarlo como un espacio socialmente producido, a la vez que dinámico y complejo. Las usuarias no eligen necesariamente para realizar sus controles establecimientos próximos a su residencia (CAPS), en oportunidades los hacen directamente en un hospital (porque consideran que allí las atienden mejor o por recomendación). Por otro lado, el acceso a los establecimientos no necesariamente depende de una distancia física; el Hospital Posadas es visto como un establecimiento próximo por su cercanía a una autopista (Acceso Oeste) y su amplia disponibilidad de servicios. 


\section{Las iniciativas municipales (o sobre esfuerzos y creatividad local)}

El nivel local adquiere relevancia a la hora del análisis de la gestión en salud en el conurbano. Los municipios tienen importantes responsabilidades en esta materia, producto del proceso de descentralización: ${ }^{13}$ están a su cargo los CAPS y algunos hospitales (responsabilidad que comparten con la Provincia y, en menor medida, con la Nación). Sin embargo, las capacidades financieras de los municipios para asumir estas responsabilidades son reducidas debido a que la transferencia de funciones no estuvo acompañada de recursos (Chiara, Di Virgilio y Ariovich, 2010).

Los entrevistados han narrado múltiples y creativas estrategias desarrolladas en el nivel municipal para hacer frente a los requerimientos locales de la atención perinatal y los problemas que atraviesan las mujeres en sus recorridos. Se destacan los casos de Morón y Moreno, donde sobresalen varios esfuerzos orientados a dinamizar y facilitar el camino de las embarazadas. En Morón, las entrevistadas mencionaron el Programa Materno Infantil Municipal que organizaba la red de obstétricas local y la atención de embarazadas y, por otro lado, dos estrategias para facilitar el acceso de las embarazadas al Hospital: el "corredor verde para las embarazadas" que reducía las colas, esperas y facilitaba el ingreso al sistema de salud, y una tarjeta que permitía viajar en colectivo gratuitamente a las mujeres que cursaban un embarazo de alto riesgo y que el personal administrativo del Hospital priorizara su atención.

En Moreno, se destacaron dos estrategias planificadas y sostenidas desde la gestión municipal, pero desarrolladas en el Hospital de la Vega (provincial), para facilitar las trayectorias de las gestantes que llegan allí por controles o estudios: 1) La Oficina de Gestión al Usuario, a donde se dirigen las embarazadas al ingresar al Hospital (allí las recibe una trabajadora administrativa municipal encargada de guiar sus recorridos: las acompaña a los consultorios y les otorga turnos); 2) El Consultorio de Orientación, encargado de recibir a las embarazadas de alto riesgo derivadas desde el primer nivel, que es atendido por dos obstétricas que controlan a la mujer y la referencian a las especialidades que su caso requiere. La intención de este dispositivo es agilizar la derivación y reducir las esperas para ser atendidas por un especialista.

Por otra parte, una propuesta que cobra gran centralidad en Ituzaingó es el "consultorio mediano y alto riesgo obstétrico", desarrollado en un CAPS debido a la ausencia de establecimientos del segundo y tercer nivel en el distrito. Además, en este municipio se destaca otro dispositivo implementado en un centro de salud municipal, llevado adelante por una médica pediatra, una obstétrica y una enfermera. Se trata de un consultorio conjunto para recién nacidos y puérperas, de demanda espontanea o con turno programado. Fue implementado para facilitar el acercamiento de las mujeres puérperas al primer nivel, quienes realizan su propia consulta mientras su bebé es controlado. Las profesionales intervinientes comentaron que el consultorio fue una propuesta de ellas y que ha costado sostenerlo (no siempre los directores del CAPS están de acuerdo en dedicar el trabajo de tres profesionales durante toda una mañana a la atención de recién nacidos y puérperas), pero que de a poco se ha ido instalando.

En Hurlingham se destaca la versión municipal del Programa "Al fin Casa”. Con el fin del financiamiento provincial se definió seguir sosteniendo el seguimiento de recién nacidos prematuros, por los logros evidenciados con la primera experiencia.

El municipio ve la efectividad que tuvo este programa. Que también tuvo que ver con la gente que trabajaba en el programa, con el compromiso de los agentes, con el compromiso de quien coordina, y vio que funcionaba, y que dejamos de tener tantos niños internados en el hospitalito, porque son todas situaciones de niños que están... si no los agarras a tiempo pueden ser muy graves. Se vio la efectividad de eso y se continuó trabajando de esa manera. No con el subsidio pero sí sigue, como si fuera el programa. [ID 29]

En síntesis, los ejemplos recorridos en este apartado habilitan a mencionar nuevamente el lugar saliente de los gobiernos municipales en la organización de la red perinatal y su dinámica, sus esfuerzos amplían el 
acceso y buscan garantizar trayectorias de continuidad. También, se destaca su autonomía relativa a la hora de delinear acciones y caminos.

\section{Los mecanismos de referencia y contrarreferencia: la informalidad mueve las redes}

El apartado profundiza sobre la referencia y contrarreferencia de usuarias y recién nacidos entre establecimientos de la red. Cabe distinguir que un actor central en estos intercambios fueron (al menos hasta el cambio de gestión nacional y provincial de 2015) los coordinadores obstétricos y neonatales ${ }^{14}$ que se desempeñabaren el nivel regional, con financiamiento nacional y en el marco de la RAP. El análisis se detiene en los instrumentos de referencia y contrarreferencia existentes, en vistas de conocer en qué medida lograron a través de la regulación de relaciones alcanzar transformaciones en el modo de organizar la atención (Chiara, 2018).

Los intercambios entre instituciones suceden en busca de mayor complejidad o por falta de camas, problemas edilicios y situaciones de paro. Aparecen menciones a la derivación de situaciones de bajo riesgo (normalmente en busca de liberar camas en establecimientos de alta complejidad), aunque no se trata de una práctica habitual. De acuerdo a lo relevado, las referencias de pacientes se pactan entre servicios y, particularmente, entre sus profesionales. Si bien los interlocutores mencionan la existencia de caminos formales, reconocen esta vía como más rápida y efectiva (aunque también informal). Sobresale que las acciones formales e informales aparecen superpuestas y no son entendidas como contradictorias.

Entre las vías formales para referir una usuaria se destacan la Región Sanitaria, el radio operador y algunas oficinas dispuestas en los establecimientos (oficinas de referencia y contrarreferencia). Si bien la intervención de la Región Sanitaria aparece como el modo correcto de derivar, no siempre interviene en la práctica. Este nivel es identificado como un actor que en ocasiones participa al momento de la referencia de una mujer, pero sus gestiones no aparecen como centrales para que pueda ser llevada a cabo (muchos intercambios se producen sin su intervención o con su participación combinada a una vía informal). También, aparecen menciones al grupo de whatsapp de los coordinadores perinatales de la Región VII (del que participan todos los jefes de servicio de obstetricia y neonatología de los hospitales), aunque se aclara que cuando hay pedidos de referencias de mujeres al alto riesgo nunca hay camas. Se destaca que, en el caso del corredor de Moreno, los actores reconocen el contacto entre servicios como formal (ya que el corredor fue así dispuesto).

Sí, recibíamos derivaciones. Y en general las derivaciones te las pide la Región Sanitaria. Siempre se pide de hospital a hospital. Se pide, levanto el teléfono y llamo a mi amigo. Eso existe y es la que mejor funciona. La formal, la Región Sanitaria pide la cama. (...) se hacen las dos cosas, la formal y la informal, y a veces hasta en paralelo: en el mismo momento que te la están pidiendo te está llamando un amigo de otro lugar. [ID 24].

El Hospital Posadas es el establecimiento de mayor complejidad en la Región VII y la referencia que todos los profesionales han mencionado al precisar la derivación de un caso de riesgo que no pueden manejar. Aunque los interlocutores de otros hospitales de nivel III han mencionado que reciben usuarias y recién nacidos derivados en busca de mayor complejidad. Además, aparecen menciones a derivaciones formales e informales a establecimientos reconocidos por su complejidad por fuera de la Región (ejemplo: al Hospital Garraham de la Ciudad de Buenos Aires).

Los interlocutores han mencionado que la circunstancia narrada provoca casos de "embudo" en los hospitales de mayor complejidad. En el Hospital Posadas se asocia a que muchas derivaciones no llegan formalmente, sino mediante el contacto de un profesional de otro servicio o, directamente, porque se les sugiere a las usuarias que se acerquen al establecimiento: "Subite al colectivo y andate al Posadas" (ID 23).

Para resolver este problema, una profesional del Servicio de Neonatología del Hospital Posadas relató que se hacen "trueques" de pacientes (bajo riesgo por alto), pero no a través de corredores habilitados sino dependientes de la decisión de los profesionales intervinientes (esta estrategia también aparece mencionada 
en otros establecimientos del tercer nivel). Asimismo, la interlocutora destaca el rol de la articulación entre el Hospital Posadas y la Maternidad Carlotto, de carácter formal, ya que descomprimió el trabajo. Esa Maternidad recibe puérperas y, también, recién nacidos para recuperación nutricional o luminoterapia. Si bien esta estrategia es exitosa, en general, los entrevistados mencionan que desde la alta complejidad cuesta referenciar a sus pacientes a un establecimiento de menor complejidad, "por si llegara a ocurrir algo en la baja complejidad" (ID 21).

La contrarreferencia de usuarios e información entre instituciones del segundo y tercer nivel es ardua y escasa y depende de la buena voluntad de los equipos. Los entrevistados explican que, por la crisis de sistema sanitario y de recursos humanos, el establecimiento que derivó después no puede recibir al recién nacido; además, se mencionó que muchas familias no quieren que sus hijos sean trasladados del lugar donde los han cuidado y contenido. En relación a la devolución de información también indican que no sucede y que depende de contactos informales (es decir, que la institución que envió el paciente llame para ver cómo sigue).

El paciente viene acá, le doy solución, después lo quiero referenciar a la zona y es muy difícil de aceptarlo. Entonces un paciente que vos seguís agudo, lo crónico sigue en su área, por más que el chico, que recibió todo, tenga un mes y tenga que estar traqueostomizado, con asistencia, tiene que estar en su área y no hay eso. Te dicen: «No tengo lugar, no tengo enfermeras, hoy no tengo médico».[ID 23]

Asimismo, la contrarrerencia al primer nivel es difícil y, según lo relevado, depende de la instalación de mecanismos de contrarreferencia (que no han sido mencionados más allá de alguna experiencia municipal específica o en los corredores destacados, Morón y Moreno). En Moreno, una profesional del primer nivel, relató que las usuarias salen del Hospital Posadas, del Hospital de la Vega o de la Maternidad Carlotto (luego del parto) con un turno en el primer nivel, que brinda la Dirección de Maternidad e Infancia municipal. "Con respecto a los otros hospitales no tenemos conexión, digamos. Dependemos de que la mujer vuelva a contarnos qué sucedió"(ID 20).

Frente a estas situaciones, aparece la pregunta acerca de los instrumentos existentes para garantizar un trabajo coordinado y la continuidad de la atención y, así, la instalación de un modelo sostenido en el trabajo regionalizado y en red. En primer lugar, interesa reflexionar acerca de los protocolos y guías de derivación. Si bien existen de un conjunto de documentos elaborados por las carteras sanitarias nacional y provincial (por ejemplo: MSAL, 2013; MS-PBA, s.f.), esto no garantizó la traducción de los lineamientos en prácticas. Los referentes de la macro-gestión consultados reconocen que se ha avanzado en la definición de protocolos de actuación y que todos los actores intervinientes conocen cómo debe procederse, pero que muchas veces actúan según normas propias. Por ejemplo, las normas respecto de cuándo derivar a las embarazadas al segundo o tercer nivel cambian según el municipio, el establecimiento o, incluso, el profesional. "Nosotros tenemos normatizado que las pacientes las derivemos a las 32 semanas al Hospital Bocalandro y a las 28, un poquito antes, al Hospital Carrillo, que son hospitales de referencia de acá del partido” (ID 26). Se destaca la excepción de los corredores de Moreno y Morón, donde los actores de la macro y meso-gestión entrevistados indican la existencia de protocolos y guías de referencia y contrarreferencia a nivel local (MEC, 2017) y los actores de la micro-gestión su uso para definir el camino de las usuarias (aunque aparecen situaciones donde el profesional define un camino distinto del formal).

Interesa hacer una mención a las hojas de referencia y contrarreferencia, particularmente en relación a la articulación entre el primer nivel y los hospitales. Las alusiones a su uso en las derivaciones varían según el nivel en que se desempeñen los interlocutores. Un profesional del Hospital Héroes de Malvinas mencionó que las mujeres nunca llegan con ella, pero que es común que los profesionales del primer nivel referencien usuarias por vías informales (un llamado, por ejemplo). También, una médica de la Maternidad Carlotto mencionó que algunas veces las mujeres llegan con un recetario con indicaciones de estudios o, también, con indicaciones verbales (difíciles de comprender). Por otra parte, las profesionales del primer nivel consultadas indican que las utilizan, aunque no siempre son hojas formales de referencia y contrarreferencia, ya que hay faltantes de este insumo en el primer nivel y se utilizan otros medios (como notas o recetarios). Algunas de 
estas interlocutoras agregan que normalmente las usuarias contrarreferenciadas vuelven sin una respuesta escrita, y esto dificulta saber cuál fue la evaluación del segundo/ tercer nivel.

Respecto a los instrumentos de referencia y contrarreferencia analizados (protocolos y guías, hojas de referencia y contrarreferencia) llama la atención su utilización discrecional y dependiente del criterio del establecimiento o del profesional. Esta situación atenta contra la coordinación, como asimismo dificulta la garantía de una continuidad de la atención en la Región estudiada, y denota una baja construcción de la red en términos formales.

Por último, interesa presentar el Mapa 4 (a continuación), que ilustra la dinámica institucionalizada de referencia y contrarreferencia (en búsqueda de una atención de mayor complejidad) entre establecimientos del segundo y tercer nivel en la red perinatal de la Región Sanitaria VII (Provincia de Buenos Aires).

Mapa 4. Dinámica institucionalizada de referencia y contrarreferencia (en búsqueda de una atención de mayor complejidad) entre establecimientos del segundo y tercer nivel. Red perinatal de la Región Sanitaria VII, Provincia de Buenos Aires.2015.

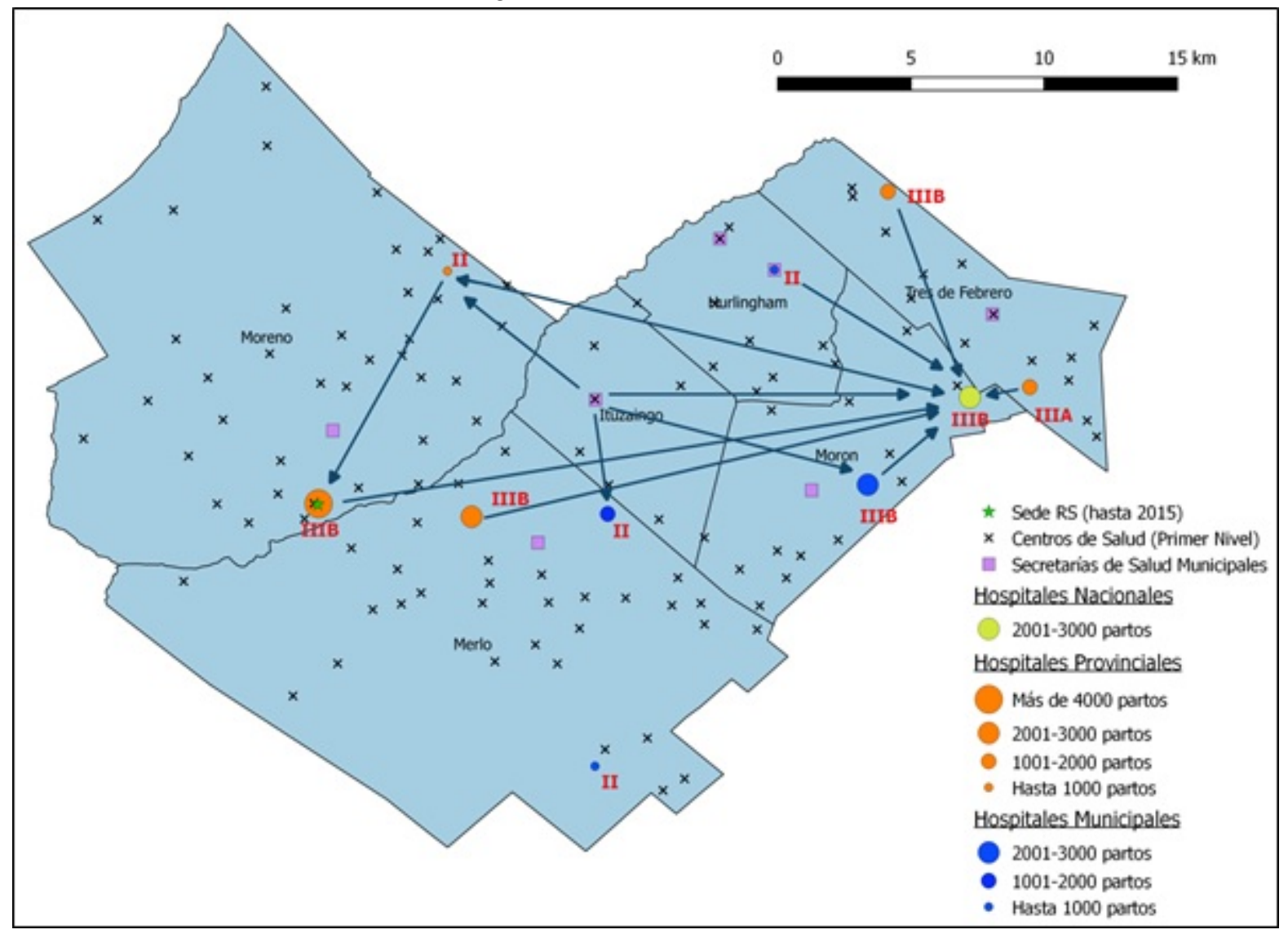

Fuente: Elaboración propia.

A primera vista se destaca que, en el Mapa 4, las flechas van en un solo sentido y siempre hacia un nivel de complejidad más alto, dado la escasa contrarreferencia formal entre instituciones (con excepción de una flecha, correspondiente al caso de la Maternidad Carlotto y el Hospital Posadas). Por otro lado, sobresale que todas las flechas confluyen en el Hospital Posadas y que prácticamente no hay articulación entre hospitales de nivel II y IIIA con otros establecimientos IIIB (sólo se evidencia una flecha en Moreno, que une a la Maternidad Carlotto con el Hospital L. y M. de la Vega). 


\section{REFLEXIONES FINALES}

En relación a la dinámica de la red y las trayectorias de las usuarias, este trabajo muestra que los recorridos se ven afectados por una multiplicidad de situaciones: el municipio de residencia, el nivel que elijan como puerta de entrada, las características específicas de cada uno de los establecimientos (que incluyen el modo en que se accede a controles, consultas y turnos). Además, las decisiones del profesional interviniente, como las de la usuaria, también imprimen características singulares a los recorridos.

Los establecimientos de segundo y tercer nivel de la red reciben personas en trabajo de parto que se acercan referenciadas desde el primer nivel, por decisión propia, luego de haber "rebotado" en otro hospital o haber sido derivadas formalmente (por falta de camas, deficiencias edilicias o falta de profesionales). También, están quienes ingresan a través de la Guardia, las UPA o el Servicio de Emergencias.

El artículo muestra que las derivaciones de usuarias de un establecimiento a otro de mayor complejidad pueden ser formales o informales (las referencias formales no deben confundirse con aquellas institucionalizadas, ya que existen situaciones informales que se institucionalizan). Sobresale que, muchas veces, la elección depende del criterio del profesional interviniente y sus vínculos personales, como de la mujer y sus posibilidades en términos de acceso. Así, los entrevistados han destacado múltiples derivaciones de pacientes que son pactadas informalmente entre servicios y sus profesionales, porque reconocen esta vía como más rápida y efectiva. Cabe agregar que las acciones formales e informales aparecen superpuestas y no son entendidas como contradictorias.

Las situaciones narradas (derivaciones formales y, sobre todo, informales) producen "embudos" en algunos establecimientos de alta complejidad. En este sentido, se destaca la dificultad de derivar pacientes del alto al bajo riesgo (con excepción de una experiencia entre el Hospital Posadas y la Maternidad Carlotto). La contrarreferencia no sólo presenta dificultades en los niveles más altos de la red, el retorno de las usuarias a los CAPS también presenta barreras.

Las negociaciones y arreglos informales aparecen como mecanismos para coordinar acciones entre niveles de atención y de gobierno y garantizar trayectorias de continuidad (no siempre con éxito). Estos arreglos refieren, especialmente donde no hay corredores formales delineados, a soluciones alcanzadas de modo informal a través de vínculos personales y favores. En relación a los instrumentos formales de referencia y contrarreferencia (protocolos y guías, y hojas de referencia y contrarreferencia), llama la atención su utilización discrecional y dependiente del criterio del establecimiento o del profesional. Este escenario atenta contra la coordinación, dificulta la garantía de una continuidad de la atención en la Región estudiada, y denota una baja construcción de la red en términos formales.

\section{REFERENCIAS}

Alonso, G. V., Gutiérrez, R. A., y Merlinsky, M. G. (2016). Federalismo y políticas municipales en la Región Metropolitana de Buenos Aires. Los desafíos de articulación interjurisdiccional en dos casos de estudio (Avellaneda y San Martín). Gestión y política pública, 25(2), 483-523.

Ariovich, A. (2015). El abordaje integral de la atención. Una aproximación a la estrategia de la atención primaria de la salud. En M. Chiara (comp.), Cuaderno de trabajo. Gestión territorial integrada para el sector salud. Los Polvorines: UNGS.

Ariovich, A. y Crojethovic, M. (2015). Las redes: un modelo organizativo para contrarrestar la fragmentación institucional del sistema de salud en la Argentina. Revista Gestión de las Personas y Tecnología, 8(24), 38-50.

Ariovich, A. y Jiménez, C. (2009). Las políticas sanitarias en el nivel local: los desempeños de la estrategia APS en tres casos del conurbano bonaerense. Ponencia presentada en las V Jornadas de Jóvenes Investigadores del Instituto de Investigaciones Gino Germani de la Facultad de Ciencias Sociales de laUniversidad de Buenos Aires, Buenos Aires. 
Ariovich, A. y Jiménez, C. (2014). Accesibilidad a la atención de la salud en el municipio de Malvinas Argentinas. Los Polvorines: Ediciones de la Universidad Nacional de General Sarmiento.

Bernztein, R., Miceli, A., Drake, I., Levcovich, M., Grau, G., Marzo, S., Pianesi, J., Borsellino, S., Atencio, A., Kamiya, M. y Ortiz, C. (2012). Implementación del Plan Operativo para la Reducción de la Mortalidad Materno Infantil, de la Mujer y de la Adolescente en Argentina, 2009-2011. Evaluación de medio término. Ministerio de Salud de la Nación.

Berra, S., Audisio, Y., Mántaras, J., Nicora, V., Mamondi, V., y Starfield, B. (2011). Adaptación cultural y al sistema de salud argentino del conjunto de instrumentos para la evaluación de la Atención Primaria de la Salud. Revista Argentina de Salud Pública, 2(8), 6-14.

Chiara, M. (2018). La salud gobernada. Politica sanitaria en la Argentina 2001-2011. Los Polvorines: Ediciones de la Universidad Nacional de General Sarmiento.

Chiara, M., Di Virgilio, M., y Ariovich, A. (2010). La coparticipación provincial por salud en el Gran Buenos Aires: reflexiones en torno a la política local (2000-2005). Salud colectiva, 6(1), 47-64.

Chiara, M., Di Virgilio, M., y Moro, J. (2009). Inequidad (es) en la atención de la salud en el gran Buenos Aires: Una mirada desde la gestión local. Postdata, 14(1), 97-128.

Fariña, D. (2018). Regionalización de la Atención Perinatal: desarrollo y propuesta. En Ministerio de Salud de La Nación, Regionalización de la Atención Perinatal en la Argentina. Barreras, experiencias y avances en el proceso de Regionalización Perinatal. Ciudad Autónoma de Buenos Aires: Ministerio de Salud de la Nación.

Gobierno de Morón. (s.f.). Centros de Salud. Recuperado de: http://www.moron.gob.ar/nuestra-gestion/salud/cent ros-de-salud-yvacunatorio/

Gutiérrez, A. (2011). Insumos para una gestión intersectorial de políticas públicas: movilidad y acceso. Territorios, 2(25), 151-172.

Lascoumes, P. y Le Galés, P. (2007). Sociologie de l'action publique (pp. 87-108). Paris: Armand Colin.

Ministerio de Salud y Desarrollo Social (MSDS) (2018). Mapainteractivo. Recuperado de http://www.desarrollosoc ial.gob.ar/mapa\#

Maternidad Estela De Carlotto (MEC). (2017). Trabajar en la Maternidad Estela de Carlotto. Guia para la organización y el funcionamiento institucional. Documento interno de trabajo.

Ministerio de Salud de la Nación (MSAL). (2010). Plan para la reducción de la mortalidad materno infantil, de las mujeres y de las adolescentes. Recuperado de http://www.msal.gob.ar/plan-reduccion-mortalidad/pdfs/plan_op erativo_reimpresion_junio2010_WEB.pdf

Ministerio de Salud de la Nación (MSAL). (2013). Recomendaciones para la Práctica del Control preconcepcional, prenatal y puerperal. Recuperado de http://www.msal.gob.ar/images/stories/bes/graficos/0000000158cnt-g02 .control-prenatal.pdf

Ministerio de Salud de la Provincia de Buenos Aires (MS-PBA). (s.f.). Guía de procedimientos para el control del embarazo y la atención del parto y puerperio de bajo riesgo. Recuperado de: http://www.ms.gba.gov.ar/sitios/tocoginecologia/2017/09/06/guia-de-procedimiento-para-el-control-del -embarazo-y-la-atencion-del-parto-y-puerperio-de-bajo-riesgo/

Ministerio de Salud de la Provincia de Buenos Aires (MS-PBA). (s. f.). Al fin en casa. Recuperado de http://www.ms .gba.gov.ar/sitios/alfinencasa/como-se-implementa-al-fin-en-casa/

Ministerio de Salud de la Provincia de Buenos Aires (MS-PBA), Programa de Apoyo a la Inversión en los Sectores Sociales de la Provincia de Buenos Aires. (2016). Fortalecimiento y desarrollo de la red perinatal de salud de la provincia de Buenos Aires.

Ministerio de Salud y Desarrollo Social de la Presidencia de la Nación. Promueven consolidar información sobre embarazo, parto, puerperio y recién nacidos. Recuperado de http://www.msal.gob.ar/prensa/index.php?option= com_content\&id=743:promueven-consolidar-informacion-sobre-embarazo-parto-puerperio-y-recien-nacidos

Municipalidad de Ituzaingó. (2014). Continúa la campaña de vacunación contra el sarampión, la rubéola y la poliomielitis. Recuperado de: https://www.facebook.com/prensa.ituzaingo/posts/612975212144910/ 
Municipio de Tres de Febrero. (2018). Centros de Salud. Recuperado de: https://www.tresdefebrero.gov.ar/salud-pu $\mathrm{blica} / \mathrm{centros}$-de-salud/

Municipio de Merlo. (s.f.). Hospitales. Recuperado de http://www.merlo.gob.ar/hospitales/

Municipio de Moreno. (2018). Unidades Sanitarias. Recuperado de http://www.moreno.gob.ar/unidades-sanitarias/

Organización Panamericana de la Salud (OPS) - Ministerio de Salud de la Nación (MSAL). (2011). Regionalización de la atención perinatal. Documentos. Recuperado de http://www.msal.gob.ar/images/stories/bes/graficos/000 0000176cnt-n02-regionalizacion.pdf

Pierini, C. (2019). Estructura y organización de una red perinatal en el conurbano bonaerense. Revista Población y Sociedad, 26(2), 34-63.

Schwarcz, A. (2011). Regionalización de la Atención Perinatal. En OPS-MSAL, Regionalización de la Atención Perinatal. Documentos.

Simioni, A., Llanos, O., Romero, M., Ramos, S., Brizuela, V. Y Abalos, E. (2017). Regionalización de la atención materna perinatal en la provincia de Santa Fe, Argentina. Revista Panamericana de Salud Publica, 41(38).

Speranza, A. M., y Kurlat, I. (2011). Regionalización del cuidado perinatal: una estrategia para disminuir la mortalidad infantil y la mortalidad materna. Rev. Argentina de Salud Pública, 2(7), 40-42.

Vilaça Mendes, E. (2013). Las redes de atención de salud. Brasil: Organización Panamericana de la Salud.

\section{Notas}

1 Refiere a los 24 municipios que rodean a la Ciudad Autónoma de Buenos Aires.

2 Se procura un lenguaje inclusivo y no sexista. Para evitar estereotipos y avanzar en una libertad dinámica (siguiendo aL. Peker) en el trabajo se intercambian femeninos, masculinos y barras.

3 Las pautas para definir el nivel de riesgo de las embarazadas, como la necesidad de su derivación al nivel correspondiente, se desarrollaban en guías nacionales y provinciales (MS-PBA, s.f.; MSAL, 2013).

4 Los servicios de obstetricia del nivel II se caracterizan por dedicarse a la atención de embarazos de bajo riesgo, la posibilidad de realizar cesáreas y no poseer terapia intensiva de adultos. Los servicios de neonatología de este nivel atienden recién nacidos mayores a 35 semanas. En el nivel III, los servicios de obstetricia atienden embarazos de alto riesgo, poseen especialidades y terapia intensiva; y los servicios de neonatología se dividen en nivel IIIA y nivel IIIB, los primeros atienden recién nacidos mayores a 32 semanas y los segundos menores de 32 semanas (Fariña, 2018).

5 En el 2010, el porcentaje de nacimientos de la Región VII sobre total provincial corresponde al 15\% y sobre el conurbano al 22\%. Las cifras refieren al sector estatal y son una elaboración propia, a partir de los datos dela Dirección de Información Sistematizada del Ministerio de Salud provincial.

6 Este programa provincial tiene como población objetivo a los recién nacidos prematuros y/o con bajo peso, y sus familias. Sus objetivos son: favorecer la detección de las alteraciones del desarrollo y los problemas de salud para iniciar precozmente la atención; promover el desarrollo de cada niño y su familia; generar entornos que prioricen el cuidado de la salud (MS-PBA, s.f.).

7 Los CIC constituyen un modelo de gestión estatal que implica, en un ámbito físico común y de escala municipal, la integración y coordinación de políticas de Desarrollo Social y Atención Primaria de la Salud (MSDS, 2018).

8 Bernztein et al. (2012, p. 52), en una evaluación del "Plan Operativo para la Reducción de la Mortalidad Materno Infantil, de las Mujeres y de las adolescentes", destacan que el conurbano tiene más de 25 establecimientos categorizados como complejos y que se estima que con 14 se podría dar respuesta a la demanda (si fueran fortalecidos en materia de recursos humanos, capacitación y estructura edilicia). Así, este diagnóstico dio cuenta de la necesidad e importancia de regionalizar la atención perinatal.

9 El apartado hace foco en el camino de las gestantes en el subsector estatal, pero la red bajo estudio, a través de los recorridos, se vincula con los subsectores privado y de las obras sociales. Las entrevistas indican que, en ocasiones, la población con cobertura de obras sociales o prepagas utiliza los servicios que ofrece la red: realiza controles o estudios, o decide tener su parto en un hospital estatal. Incluso, se indicaron derivaciones de establecimientos públicos a privados y viceversa.

10 Estos controles permiten un seguimiento de la salud de la mujer, el asesoramiento en temas de vacunación (y la facilitación de su aplicación), la promoción de la lactancia y la planificación familiar (a través de la asesoría acerca de los métodos anticonceptivos disponibles y su entrega). 
11 "El SIP es una aplicación desarrollada por el Centro de Perinatología y Desarrollo Humano de la Organización Panamericana de la Salud/Organización Mundial de la Salud (OPS/OMS) con el objetivo de recopilar información relacionada con la atención del embarazo, el parto, el puerperio y los recién nacidos" (Ministerio de Salud y Desarrollo Social de la Presidencia de la Nación, 2018).

12 En el conurbano, la fragmentación y la segmentación del sistema sanitario, como también la desigualdad de los territorios, han provocado un desigual acceso a la salud por parte de la población, que en un 39,4\% depende de la red estatal (Censo 2010). Cabe aclarar que quienes demandan los servicios del sector público son mayormente personas o familias de bajos ingresos que carecen de otro tipo de cobertura. Se destaca que la fragmentación y segmentación redundaron en una débil y desarticulada construcción de la atención perinatal pública, que hizo mella en los indicadores de morbi-mortalidad materna e infantil (para fines de los 2000), situación que motivó el trabajo en RAP y mi interés en las redes perinatales.

13 Esta estrategia, que acompañó la reforma democrática, se caracterizó por una transferencia de funciones de arriba hacia abajo, para garantizar la eficiencia y disminuir las presiones fiscales del nivel nacional, que no estuvo acompañada de una transferencia de recursos. En la Provincia de Buenos Aires, la descentralización operó fuerte sobre los municipios, que asumieron nuevas responsabilidades, en muchas ocasiones no establecidas formalmente. A esta situación se agregó la ausencia de una definición respecto de los niveles a ser cubiertos por cada unidad territorial (Chiara, Di Virgilio y Moro, 2009).

14 Estos coordinadores, cercanos al territorio y a los establecimientos, conocían la situación de camas, intervenían en la referencia y contrarreferencia y en situaciones críticas. 